\section{Protective transcriptional repression by junctophilin 2 fragment}

Junctophilin 2 is a structural protein that connects the transverse tubule membrane to the sarcoplasmic reticulum (SR) membrane in microdomains known as cardiac dyads. A new study published in Science now shows that a cleaved fragment of the junctophilin 2 $\mathrm{N}$-terminus (JP2NT) translocates to the nucleus and has cardioprotective effects through regulation of gene transcription.

The cardiac dyad is the site of $\mathrm{Ca}^{2+}$ entry into cardiomyocytes via voltage-gated L-type $\mathrm{Ca}^{2+}$ channels (LTCCs), which in turn triggers $\mathrm{Ca}^{2+}$-induced $\mathrm{Ca}^{2+}$ release from the $\mathrm{SR}$ via the ryanodine receptor 2 (RYR2). Junctophilin 2 is important for establishing and maintaining the $12-15 \mathrm{~nm}$ gap between the sarcolemma and SR membranes in the cardiac dyad. Junctophilin 2 cleavage by calpain in response to cardiac stress causes excitation-contraction uncoupling, leading to heart failure progression.

Moreover, the JP2NT fragment generated by junctophilin 2 cleavage has now been shown to have DNA-binding properties. JP2NT translocates to the nucleus, binds to chromatin via an evolutionarily conserved binding domain and competes with the transcription factors MEF2 and TATA-binding protein. As a result, transcription of genes related to cell growth, hypertrophy, inflammation and fibrosis is repressed.

Mice with cardiac-specific overexpression of JP2NT had better cardiac function in response to pressure-overload than control mice. Conversely, mice with a loss-offunction mutation that disables the nuclear translocation of JP2NT had more severe cardiac hypertrophy and worsened heart function in response to pressure-overload than wild-type littermates.

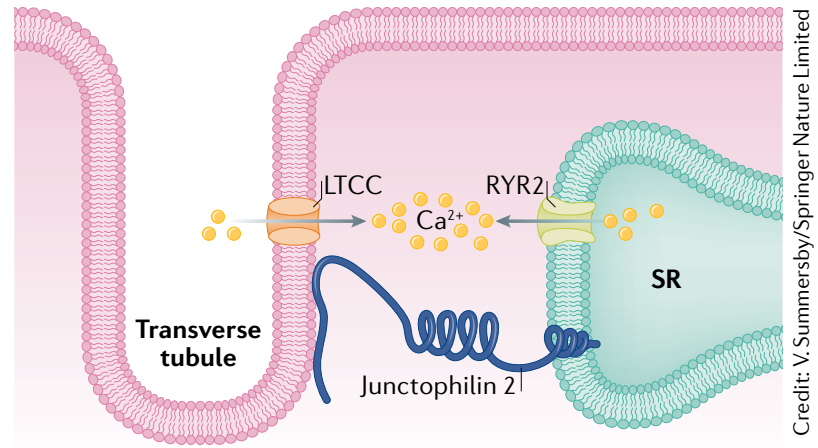

"These new findings reveal a previously unknown self-protective mechanism that enables failing cardiomyocytes in the stressed myocardium to transduce mechanical information into salutary transcription reprogramming," comments Long-Sheng Song, corresponding author of the article. "We plan to use a gene therapy approach to investigate the beneficial effects of delivering JP2NT or functional peptide in preclinical animal models of heart failure."

Gregory B. Lim

protective effects

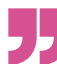

\title{
CARDIOMYOPATHIES
}

\section{Inflammation linked to Takotsubo}

Acute stress-induced (Takotsubo) cardiomyopathy is typically associated with a major stressful event and has a similar presentation to that of acute myocardial infarction, but its precise pathogenesis is unclear. A new study shows that this syndrome is characterized by macrophage infiltration in the myocardium, changes in circulating monocyte subsets and increased levels of pro-inflammatory cytokines in the blood, with some of these changes persisting for $\geq 5$ months. "This study demonstrates for the first time that Takotsubo cardiomyopathy is accompanied by myocardial and systemic inflammatory activation," says lead investigator Dana Dawson.

The study included 55 patients with acute Takotsubo syndrome (mean age 64 years; $90 \%$ women) and 51 matched control individuals. To examine macrophage infiltration in the myocardium, Dawson and colleagues combined cardiac MRI with intravenous infusion of ultrasmall superparamagnetic particles of iron oxide (USPIO), which are phagocytosed mainly by activated macrophages. Systemic inflammation was measured by assessing monocyte subpopulations and cytokine levels in blood.

Myocardial uptake of USPIO was higher in patients in the acute phase of Takotsubo than in control individuals, suggesting a macrophage-driven cellular infiltration in the myocardium. Patients with acute Takotsubo also had elevated levels in the blood of the pro-inflammatory cytokines IL-6, IL-8 and CXCL1, an increase in pro-inflammatory monocytes $\left(\mathrm{CD} 14^{++} \mathrm{CD} 16^{-}\right)$and a decrease in intermediate $\left(\mathrm{CD} 14^{++} \mathrm{CD} 16^{+}\right)$ and nonclassical (CD14 ${ }^{+}$CD $16^{++}$) monocytes. USPIO enhancement was no longer detectable after 5 months of the index event, but some of the systemic changes persisted, such as increased IL-6 levels and decreased number of intermediate monocytes.
ORIGINAL ARTICLE Guo, A. et al. E-C coupling structural protein junctophilin-2 encodes a stress-adaptive transcription regulator. Science https://doi.org/10.1126/science.aan3303 (2018)

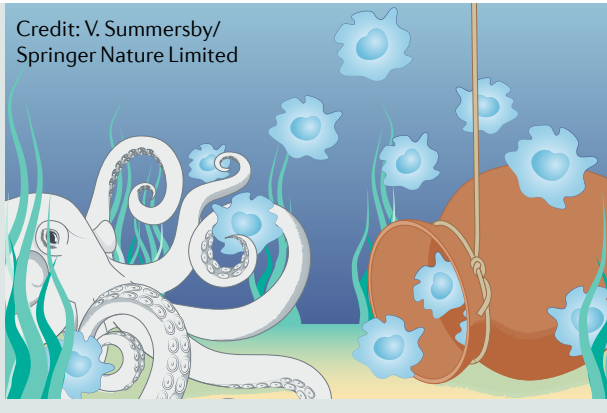

"These findings demonstrate that the acute inflammation evolves into a low-grade, chronic inflammatory state, which explains the lack of functional recovery seen in a proportion of patients with Takotsubo syndrome," remarks Dawson. "It is, therefore, imperative that we search for therapeutic strategies to mitigate the clinical consequences of this condition and prevent recurrences," she concludes.

Irene Fernández-Ruiz

ORIGINAL ARTICLE Scally, C. et al. Myocardial and systemic inflammation in acute stress-induced (Takotsubo) cardiomyopathy. Circulation https://doi. org/10.1161/CIRCULATIONAHA.118.037975 (2018) FURTHER READING Kastaun, S. et al.

Psychosocial and psychoneuroendocrinal aspects of Takotsubo syndrome. Nat. Rev. Cardiol. 13, 688-694 (2016) 